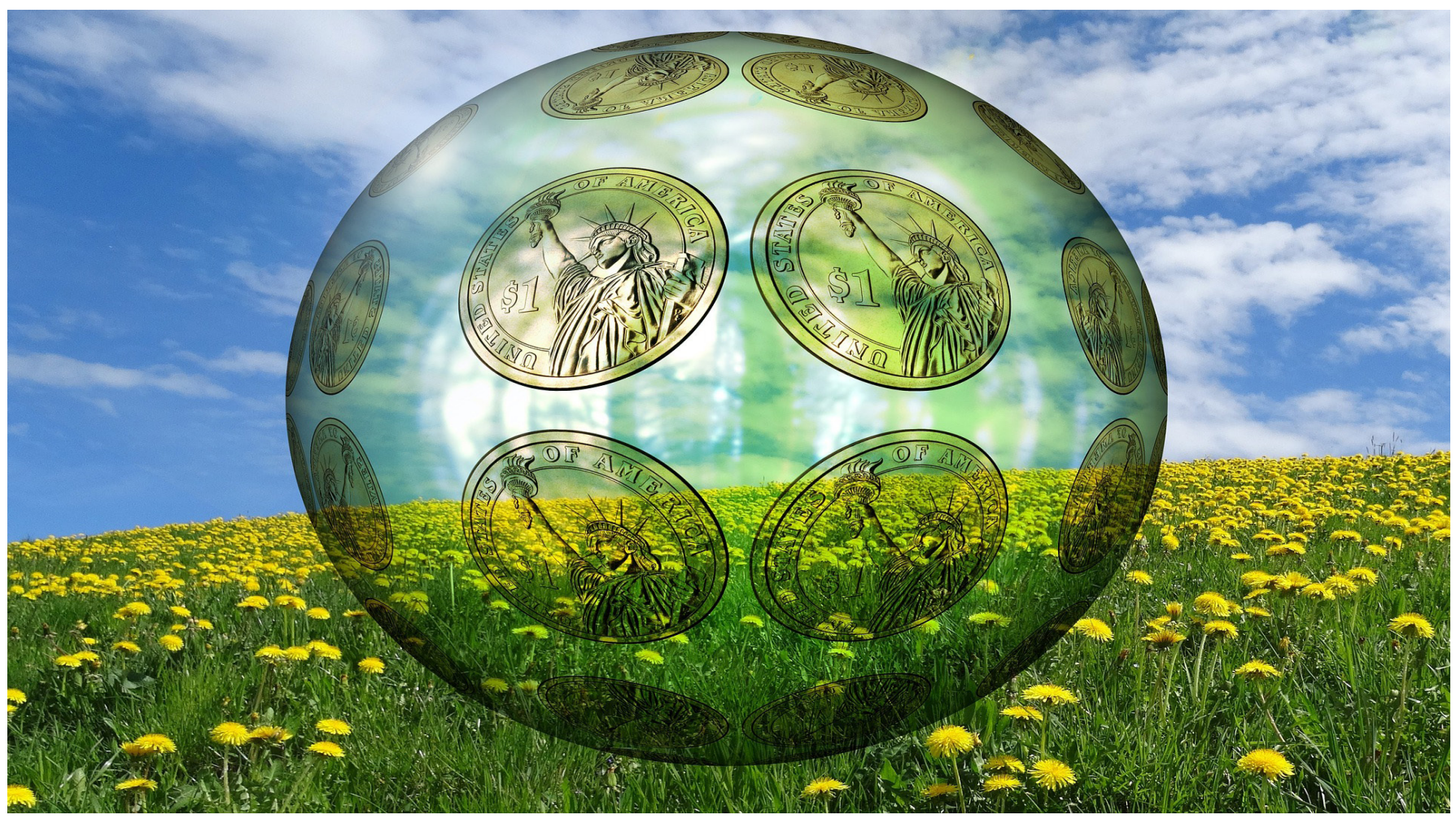

\title{
Identificación de estrategias de gestión pública para el fomento del turismo sostenible en Chinácota, Norte de Santander
}

Economic situation of the households by Covid Identification of public management strategies to promote sustainable tourism in Chinácota, Norte de Santander

Liliana Leguizamón-Gámez

Especialista en Gestión Pública,

Fundación de Estudios Superiores Comfanorte, Cúcuta, Colombia

Nefer Leandro Mora-Durán

Especialista en Gestión Pública,

Fundación de Estudios Superiores Comfanorte, Cúcuta, Colombia
Walter Omar Pabón-Ramírez

Especialista en Gestión Pública,

Fundación de Estudios Superiores Comfanorte, Cúcuta, Colombia

Carolina Marulanda-Ascanio

Especialista en Economía

Fundación de Estudios Superiores Comfanorte, Cúcuta, Colombia 
Cómo citar: Leguizamón-Gámez, L., Mora-Durán, N.L., Pabón-Ramírez, W.O. \& Marulanda-Ascanio, C. (2020).

Identificación de estrategias de gestión pública para el fomento del turismo sostenible en Chinácota, Norte de

Santander. Reflexiones contables (Cúcuta), 3(2), 29-39.

\section{Resumen}

Este trabajo tiene como propósito identificar factores que se puedan desarrollar en cuanto al turismo sostenible en la región teniendo como punto de partida la gestión pública como eje central para que se puedan formular proyectos de inversión, se busca identificar la oferta turística del municipio de Chinácota, la percepción de los visitantes y de esta manera tener una idea clara de sobre cómo se podría fortalecer el municipio económicamente través del turismo con enfoque de responsabilidad de sostenibilidad teniendo en cuenta el (plan de desarrollo nacional 2018-2022) y los demás decretos que se están generando para este sector, Así pues, la investigación busca detallar de manera cuantitativa y descriptiva, basado en la opinión de los habitantes, las características y ventajas de la región; el establecimiento de estrategias pueden adoptarse para impulsar el turismo sostenible en el municipio de Chinácota en 2020, instituyendo e identificando las necesidades, fortalezas y posibles cambios para la mejora progresiva y perdurable del turismo en la región de Chinácota en Norte de Santander, obteniéndose de manera concluyente que existe factores de deficiencia de tipo personal, cultural, y organizacional, así como factores coyunturales como la actual pandemia ocasionada por el Covid-19, que inciden de manera notoriamente desfavorable en el impulso del turismo en el municipio de Chinácota Durante la anualidad en curso

Palabras clave: turismo sostenible, gestión pública

\section{Abstract}

The purpose of this work is to identify factors that can be developed in terms of sustainable tourism in the region, having as a starting point the public management as a central axis so that investment projects can be formulated, the perception of visitors and thus have a clear idea of how the municipality could be economically strengthened through tourism with a focus on sustainability responsibility, taking into account the (national development plan 2018-2022) and other decrees that are being generated for this sector. Thus, the research seeks to detail in a quantitative and descriptive manner, based on the opinion of the inhabitants, the characteristics and advantages of the region; the establishment of strategies can be adopted to promote sustainable tourism in the municipality of Chinácota in 2020, instituting and identifying the needs, strengths and possible changes for the progressive and lasting improvement of tourism in the Chinácota region in Norte de Santander, It has been conclusively obtained that there are personal, cultural and organizational factors of deficiency, as well as economic factors such as the current pandemic caused by the Covid-19, which have a notoriously unfavorable impact on the promotion of tourism in the municipality of Chinácota.

Keywords: sustainable tourism, public management 


\section{Introducción}

El desarrollo de la presente investigación, versa sobre identificación de estrategias de gestión pública para el fomento del turismo sostenible en Chinácota, Norte de Santander, estructurada primeramente desde el análisis general de información existente sobre el tema, desde lo cual se desciende a denotar la importancia del desarrollo turístico en Colombia como línea estratégica del gobierno nacional; examinando de manera subsiguiente la relevancia del turismo en la región.

Norte de Santander al ser el Departamento fronterizo más importante del país, con la mayor capacidad de intercambio económico y cultural, derivado de su legado histórico; además de contar con una ostensible existencia de recursos minero energéticos, riqueza paisajística, fortalezas agrícolas y ganaderas, cuenta con la posibilidad de aprovechamiento y enfoque de estos factores para promover estrategias que originen el impulso del turismo sostenible en su territorio y particularmente la municipalidad de Chinácota objeto de la investigación.(Pardellas2004)

El turismo visto como un potencial económico es la opción que los dirigentes públicos y líderes deben fortalecer pero que hasta el momento en la región no se ha desarrollado tampoco se ha tenido en cuenta más sin embargo el municipio es visto como destino turístico, prestando así diversos servicios desde la tradición, la necesidad de suplir la demanda de todos aquellos que frecuentan la zona, es por ello que se apremiante construir y fortalecer bases sólidas con respeto a este sector ya que se cuenta con un amplio panorama respecto de paisaje, clima, gastronomía, historia para hacer de este municipio un ejemplo en proyectos sostenibles desde el turismo.

Por lo tanto el objetivo de esta esta investigación ha sido la identificación de la oferta turística del municipio donde se analizaron capacidad hotelera, estado de las vías, percepción de los turistas, estado de vías, poder adquisitivo entre otros. De otra parte se buscaba identificar estrategias que se puedan establecer desde la gestión pública como fin de este proyecto y que a futuro se generen propuestas aterrizadas con capacidad de llevar al desarrollo del sector turístico en el municipio con un propósito de sostenibilidad.

Según cifras del Ministerio de Comercio, Industria y Turismo 2018 entre 2011 y 2017, en Colombia se presentaron destacados crecimientos en los principales indicadores del turismo en el país: se incrementó en un $69 \%$ las llegadas de viajeros internacionales, $52 \%$ la generación de divisas, 19\% la creación de nuevos empleos y $74 \%$ en la incursión de nuevas empresas al sector, generando una oferta presente en alrededor de 281 municipios del territorio nacional. De igual forma, según el Departamento Nacional de Estadística (DANE), el turismo, medido desde la rama de hoteles y restaurantes, representó el 3,78\% del Producto Interno Bruto (PIB) en Colombia durante 2017 (Ministerio de Comercio, Industria y Turismo, 2018), evidenciando su gran potencial de desarrollo.

Este contexto, hace que el sector turismo sea un renglón estratégico para el Gobierno Nacional, considerado como "el nuevo petróleo de Colombia", una opción viable y rentable de desarrollo sostenible donde el país, basado en su diversidad biológica y cultural, fortalece su competitividad como una alternativa para la generación de divisas, para crear empleo y mejorar las condiciones de vida de la población; así como constituirse en una iniciativa para la transformación regional, la reconciliación entre los colombianos y mejorar la imagen del país.

El turismo es una de las actividades económicas más importantes del planeta, representa 30\% de las exportaciones en el comercio global de 
servicios, generando 1 de cada 11 empleos y el $10 \%$ del PIB mundial UNTWO (2017) De acuerdo con esta organización, en 2017 se movilizaron 1332 millones de viajeros en todo el mundo y el turismo tiene una perspectiva de crecimiento anual hasta el 2030 cercana a 3,8\%; no obstante, para los destinos turísticos de las economías emergentes, como Colombia, este crecimiento ha sido superior al promedio global, representando una variación de 4,8\% promedio anual entre 2005 y 2017.

El Departamento cuenta con importantes recursos mineros como petróleo, carbón, calizas y metales preciosos, así como con zonas agrícolas y ganaderas con una considerable producción de arroz, caña, palma de aceite, ganado vacuno y caprino entre otros, que han sido poco tecnificadas y explotadas a escala industrial como producto de la histórica influencia subversiva, que limita la inversión en tecnificación y transporte de los productos, especialmente en sus provincias del Catatumbo y Ocaña ubicadas en la parte norte del Departamento. Además de sus riquezas naturales, Norte de Santander concentra un amplio legado histórico y cultural apenas mencionado, que a diferencia de otros destinos turísticos del país como Bogotá, Cartagena y Popayán que atraen anualmente a numerosos turistas cuya motivación es rescatar la historia y tradición cultural de Colombia, son relativamente desconocidos para los nativos de la región así como buena parte de la historia que la ciudad y el Departamento han aportado al desarrollo nacional. (Moreno 2014).

Estos insumos naturales y culturales conforman una amplia oferta turística departamental como da cuenta el Fondo Mixto de Promoción Turística Departamental-que sumada al marco normativo de apoyo económico de proyectos turísticos de desarrollo socioeconómico de las regiones, y al nuevo marco de reconciliación nacional subsecuente al proceso de paz con las Fuerzas Armadas Revolucionarias de
Colombia (FARC) y el Ejército de Liberación Nacional), (ELN), favorecen la articulación del plan de desarrollo nacional y departamental con la inversión pública y privada, para generar proyectos de explotación turística responsable y socialmente comprometida, que además de propiciar el desarrollo social y económico de la región fundamenten el turismo histórico, cultural, de aventura y natural, como nueva impronta de una región en proceso de transformación y con una prometedora proyección económica en el mediano plazo (Guerrero y Espinoza, 2017).

Estos insumos naturales y culturales conforman una amplia oferta turística departamental como da cuenta el Fondo Mixto de Promoción Turística Departamental-que sumada al marco normativo de apoyo económico de proyectos turísticos de desarrollo socioeconómico de las regiones, y al nuevo marco de reconciliación nacional subsecuente al proceso de paz con las Fuerzas Armadas Revolucionarias de Colombia (FARC) y el Ejército de Liberación Nacional), (ELN), favorecen la articulación del plan de desarrollo nacional y departamental con la inversión pública y privada, para generar proyectos de explotación turística responsable y socialmente comprometida, que además de propiciar el desarrollo social y económico de la región fundamenten el turismo histórico, cultural, de aventura y natural, como nueva impronta de una región en proceso de transformación y con una prometedora proyección económica en el mediano plazo (Guerrero y Espinoza, 2017).

Chinácota, conformado por aproximadamente 16.350 personas y un total de 7618 viviendas habitables (DANE, 2018), no solo cuenta con sus bellos paisajes, su cultura religiosa, sus platos típicos también está ubicado en límites de zona protegidas como el parque el TAMA también posee valiosos recursos y caudales hídricos como la quebrada Iscalá que desemboca en el rio Pamplonita, de donde 
surten de agua los habitantes de la capital del departamento Norte de Santander. Por lo anterior es un municipio que posee diversas características lo que lo proyecta a ser sostenible turísticamente.(Matagira 2012).

Algunos de los servicios turísticos más buscados son esparcimiento, parques temáticos, gastronomía, descanso, alojamiento, caminata, visita a monumentos religiosos turismo histórico entre otros; demanda que el Municipio no ha suplido, a pesar de tener 18 hoteles, 31 restaurantes (Guerrero 2017), al igual que 33 atractivos turísticos dentro de los que se encuentran; cerros, lagunas, parques naturales, senderos ecológicos, iglesias, lugares deportivos entre otros, para disfrutar en familia y amigos). Por otra parte, este incremento de visitantes también ha ocasionado que la ocupación de suelo se haya venido manejando sin planeación alguna, afectando de esta manera las zonas aledañas a los caudales, siendo este uno de los factores causales del déficit de agua del servicio de acueducto en temporada de festivos como puentes, semana santa, fin de año, ferias, entre otros.

Lo anterior ha venido inquietando a la comunidad en general, viendo la necesidad de tomar medidas de mejora en la economía a través del turismo pero con proyectos sostenibles realizados desde los entes gubernamentales. El servicio turístico prestado no es el más adecuado, debido a que lo realizan sin procesos establecidos en los planes de desarrollo, es decir legalmente establecidos, siendo prestados por los mismos habitantes de las veredas sin capacitación, debido al gran auge al que sea abocado el municipio en la zonas rurales y vieron la oportunidad de ofrecer variedades gastronómicas y de esparcimiento, aunque también se debe recalcar que se encuentran algunos establecimiento muy bien organizado.
Para esta investigación se planteó como objetivo identificar estrategias de gestión pública para el fomento del turismo sostenible en Chinácota, Norte de Santander, 2020.

\section{Gestión pública local}

Cuando se habla de gestión Pública se hace énfasis en la forma como los actos se vuelven eficientes, eficaces y en el momento que se haga evaluación de los mismos, estos sean verificables y cumplan los objetivos propuestos. Partiendo de lo anterior la administración local debe concebir a la entidad territorial como una organización que busca los mayores niveles de bienestar y de servicios sociales para su población. En consecuencia, el alcalde debe ser un líder que asume funciones como planificador, organizador y controlador de la organización que dirige. En este orden de ideas, en el municipio se deben tomar decisiones, con base en los objetivos, estrategias, y políticas priorizadas en el proceso de planificación; orientar la ejecución de las acciones planeadas, y deben llevar a cabo evaluaciones de las acciones adelantadas, con el fin tanto de dar cuenta a la comunidad y a los órganos de control, como de tomar correctivos. El concepto que reúne estos elementos - planeación, ejecución, seguimiento y evaluación para cumplir con las competencias y alcanzar objetivos y metas de desarrollo acordadas democráticamentees el de gestión pública integral orientada a resultados, objeto central del presente documento (Cruz 2009).

\section{Desarrollo turístico sostenible.}

Lo importante de generar desarrollo sostenible en las poblaciones, partiendo del turismo es lograr contribuir al mejoramiento social, económico y cultural, mostrando los atractivos turísticos y pobladores que allí existen. Según la Organización Mundial del Turismo, "las directrices para el desarrollo sostenible del turismo y las prácticas de gestión sostenible 
son aplicables a todas las formas de turismo en todos los tipos de destinos, incluidos el turismo de masas y los diversos segmentos turísticos. Los principios de sostenibilidad se refieren a los aspectos ambiental, económico y sociocultural del desarrollo turístico, habiéndose de establecer un equilibrio adecuado entre esas tres dimensiones para garantizar su sostenibilidad a largo plazo (Ministerio de Comercio, Industria y Turismo, 2009) igualmente el turismo se caracteriza por ser una actividad social y económica relativamente joven y por englobar a una gran variedad de sectores económicos y disciplinas académicas (Morillo 2011).

"turismo sostenible", este se vuelve primero un promotor de la conservación de tal patrimonio cultural y natural, si es establecido de forma planificada, integrada, abierta, dimensionada y participativamente, es entonces viable y seguramente duradero en el tiempo, ahora sí se puede hablar de turismo sostenible como una alternativa de desarrollo comunitario, el turismo sostenible sería el detonante, el dinamizador del sitio de interés al ponerlo en valor, una vez puesto en valor despierta interés ya no solo en antropólogos, arqueólogos y etnógrafos, ahora despierta interés en los turistas, pero esto no basta, los líderes y demás personas participes de la actividad turista del sitio de interés deben enfocar sus esfuerzos de promoción y comercialización en los segmentos de mercado interesados del patrimonio cultural (Bertony 2008).

Varios destinos turísticos comunitarios dependen netamente de los ingresos percibidos por llegadas de turistas, lo que involucra un trabajo más exhaustivo en torno a la preservación del patrimonio, esto en muchos casos los hace destinos más exclusivos y caros, en relación a un destino turístico comunitario que percibe réditos del estado y que es capaz de montar toda una estructura que facilite la visita, promoción y comercialización del destino, entonces la autogestión se vuelve un aspecto importante del destino, los comuneros deben buscar mecanismos que les permitan montar toda ese andamiaje de forma similar a un destino comunitario que recibe réditos del estado, pero todo esto a través de autogestión, no resulta fácil, pero a través del diálogo, planificación y socialización (Reyes et al, 2017). Todo lo anterior se puede expresar en beneficios sin necesidad de sobre estimar los costes y demás impuestos para el desarrollo del turismo sostenible de esta forma cumplir los objetivos de sostenibilidad.

\section{Desarrollo Turístico Del Municipio De Chinácota.}

El municipio se ha constituido en un centro turístico de cabañas de descanso para los habitantes del área metropolitana de Cúcuta, que buscan clima y paisaje para su recreación. La oferta turística está representada en, aproximadamente, 200 cabañas en el 2007 "superando en estos tiempos las 400 cabañas, construidas y un potencial de visitantes, los fines de semana (4 sábados y 4 domingos por mes), de 11.093 vehículos-mes, con un número aproximado de 4 personas por vehículo para un total de 44.372 turistas (equivalente a 5.547 turistas por fin de semana). Igualmente, cuenta con los atractivos turísticos del Páramo Mejué y la riqueza histórica de la Hacienda Iscalá, con una bella casa colonial que perteneció al Presidente de Colombia, General Ramón González Valencia; la casa colonial donde se firmó el pacto de paz de la guerra de Los Mil Días, la parroquia San Juan Bautista, la Plaza de Toros y otra casonas coloniales, ubicadas en el entorno urbano del Municipio.(Ramírez et al 2011). 


\section{Método}

La investigación presenta un enfoque cuantitativo que según Hernández, Fernández y Baptista (2014, p. 4) representa un conjunto de procesos secuenciales y probatorios. Cada etapa precede a la siguiente y no es posible saltar o eludir pasos. Finalmente acompañada de metodología del marco lógico puesto que la Metodología de Marco Lógico es una herramienta para facilitar el proceso de conceptualización, diseño, ejecución y evaluación de proyectos.

El énfasis del Marco Lógico está centrado en la orientación por objetivos, la orientación hacia grupos beneficiarios y el facilitar la participación y la comunicación entre las partes interesadas. Es una investigación de carácter descriptivo en el que se analizarán las ventajas de la región al igual que lo que opinan sus habitantes. Se establece descriptiva ya que como dice Bernal (2000) es un nivel básico de investigación y se convierte en base para otras clases de investigación y se soporta principalmente en técnicas como la encuesta, la entrevista, la observación y la revisión documental.

Al desconocer el número de personas se tomará una muestra representativa probabilística para poblaciones infinitas, la cual se aplicará y se trabajará con la muestra que arroje el análisis. La fórmula es la siguiente:

\section{Valores:}

$\mathrm{n}=$ muestra

$Z=$ nivel de confianza

$\mathrm{p}=$ probabilidad del éxito

$\mathrm{q}=$ probabilidad de fracaso

$\mathrm{E}=$ Margen de error

Utilizando un nivel de confianza del $95 \%$, un margen de error del $5 \%$, una probabilidad del éxito del $50 \%$ y probabilidad de fracaso del $50 \%$ la muestra representativa seria la siguiente:

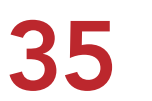

El número total de pobladores que conforman la muestra representativa es de 384 personas.

Para describir la oferta turística del municipio de Chinácota y su contribución en la sostenibilidad económica y financiera se realizará una entrevista estructurada a la dependencia de cultura y turismo del municipio donde se verifique estadística y datos importantes del desarrollo turístico y cultural de la región. Para este proceso se realizará un cuestionario estructurado y se plasmará la información obtenida en una matriz documental.

Para la recolección de información se hizo uso de instrumentos cuantificables como la encuesta y métodos descriptivos como la entrevista y revisión de documentos, el proceso de encuesta arrojo 384 respuestas a 11 preguntas previamente avaladas por experto estadístico y se realizó proceso de entrevista al encargado de la Secretaria de agricultura desarrollo económico y turismo Chinácota.

\section{Resultados}

\section{Descripción de la Oferta Turística del Municipio de Chinácota}

En esta primera fase de identificación de la oferta turística del Municipio se adaptó la información suministrada por la secretaria de agricultura desarrollo económico y turismo a fecha de septiembre de 2019 de Chinácota Norte de Santander, información que no ha sido actualizada y aunque cuentan con bases de datos con fechas, fotografías, tipo de servicio que prestan, capacidad de atención denota que apremia darle la importancia al turismo iniciando con la creación de una secretaria exclusivamente encargada de este sector. (Chavez et al 2013). 
Identificación de estrategias de gestión pública para el fomento del turismo sostenible en Chinácota, Norte de Santander

36

Cuadro 1. Relación de entidades encuestadas

\begin{tabular}{|l|c|c|}
\hline Establecimientos comerciales & Cantidad & Capacidad de atención \\
\hline Restaurantes & 4 & 74 \\
\hline Comidas rápidas & 10 & 300 \\
\hline Venta de postres & 7 & 1153 \\
\hline Atractivos turísticos & 33 & 3000 \\
\hline \multicolumn{1}{|c|}{ TOTAL } & 54 & 4527 \\
\hline
\end{tabular}

Fuente: Los investigadores

\section{Análisis de Precepción de los Ciudadanos Frente a la Oferta Turística del Municipio}

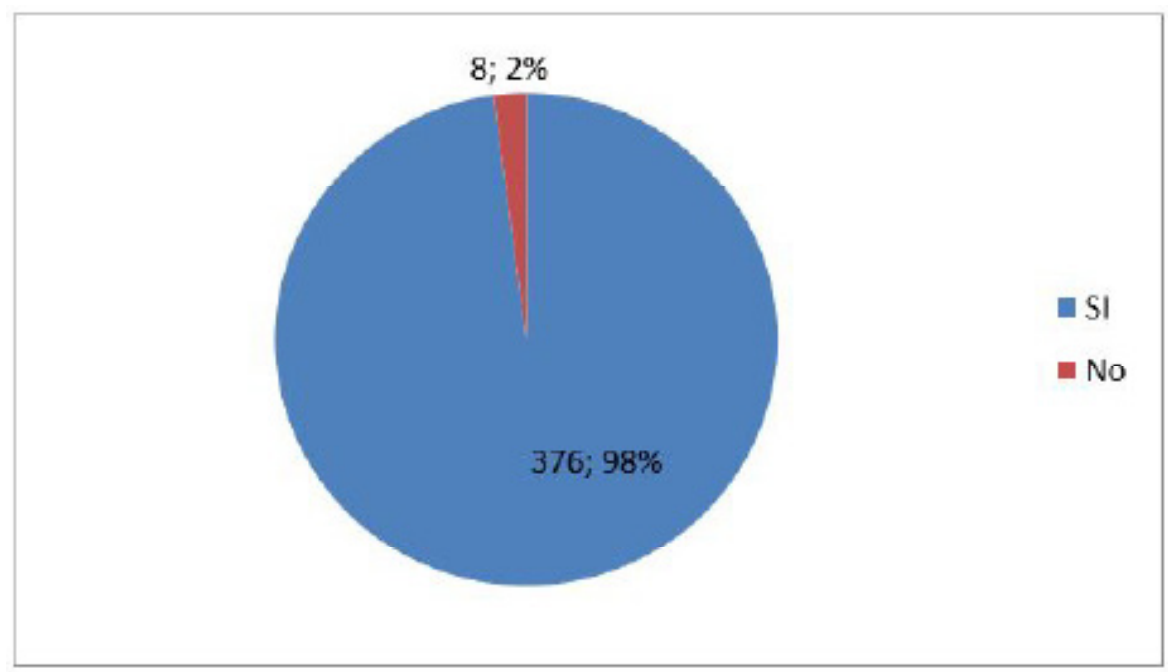

Figura 1. Intenciones o deseos de visita

Fuentes: los investigadores

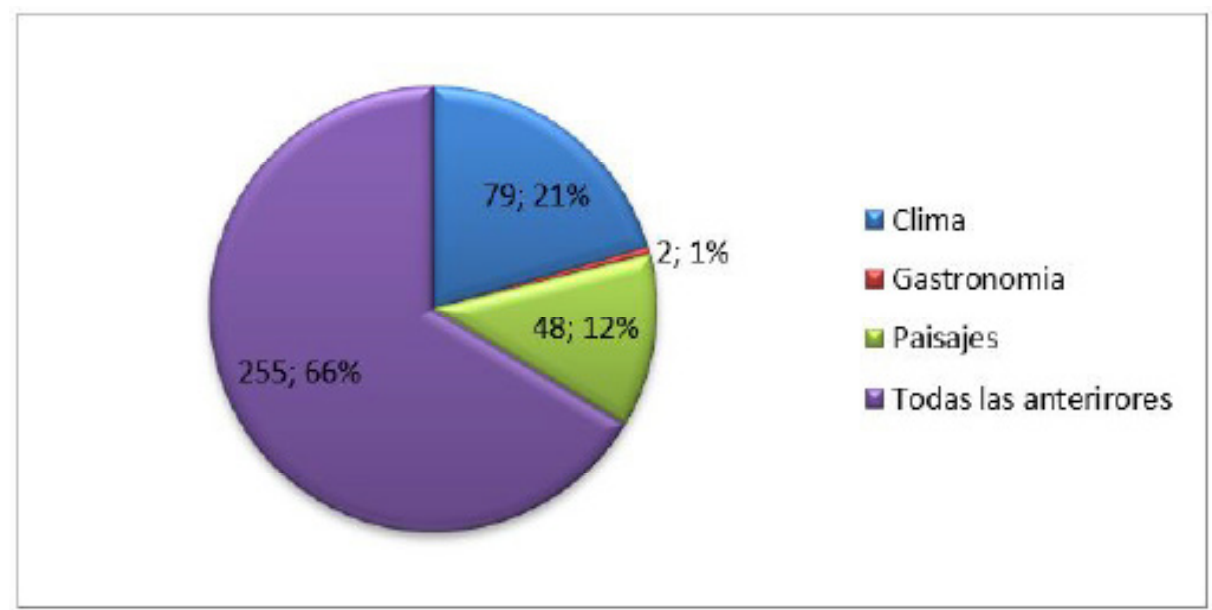

Figura 2. Motivos para ir a Chinácota

Fuentes: los investigadores 


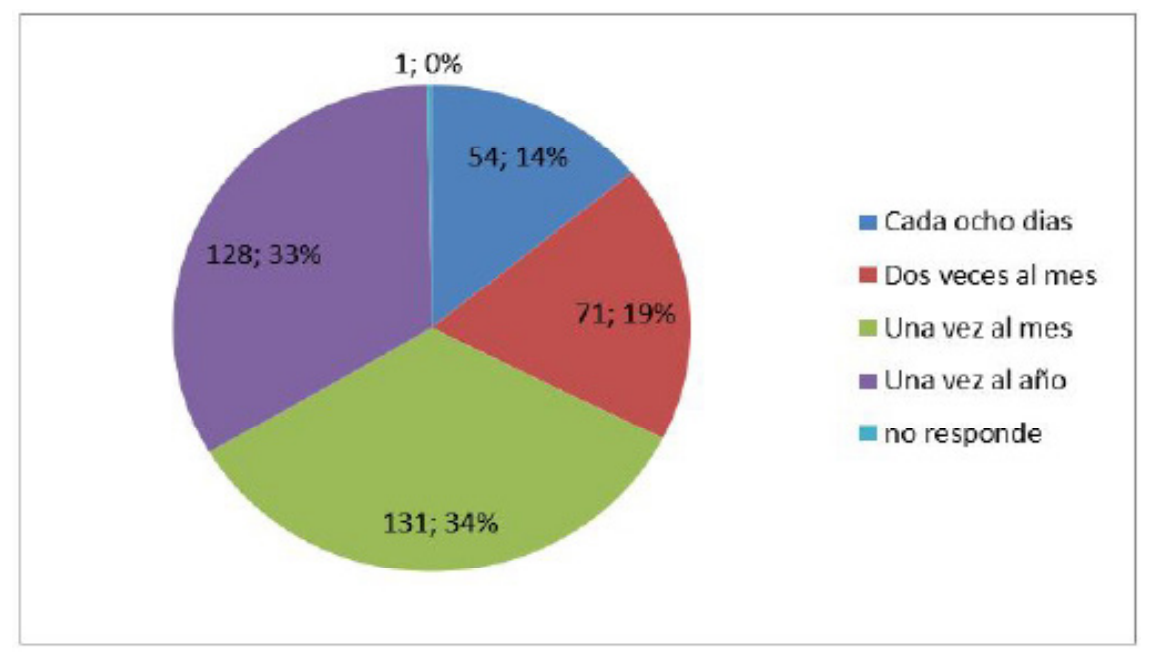

Figura 3. Frecuencias de visita

Fuentes: los investigadores

En las tres figuras anteriores vemos claramente la percepción del turista con respecto a todo lo que tiene por ofrecer el municipio de Chinácota con respecto a servicios turísticos, brinda una idea clara que cuenta con lo más importante que puede buscar una región que se desea orientarse hacia el turismo como lo es la taza de intención de visita de un $98 \%$ seguido por las inclinaciones o preferencias a la hora de visitar el municipio siendo estas su gastrómana sus paisajes y su agradable clima, a esto le agregamos que la frecuencia de visita es constante. De acuerdo a la información recolectada en esta investigación de la cual surgen las bases sólidas para identificar estrategias de gestión pública para el fomento del turismo sostenible en el municipio de Chinácota

\section{Conclusiones}

Enfocar la gestión pública en manejos de recursos que contribuyen a satisfacer las necesidades sociales, económicas y ambientales de la región respetando los recursos hídricos, ecológicos, diversidad biológica, costumbres ancestrales e identidad de su patrimonio cultural mediante la creación de un plan decenal de turismo que se pueda conectar con los de ordenamiento nacional y departamental donde se puedan obtener recursos de forma directa ya que se evidencia que los recursos para inversión turística son nulos en el municipio.

Incluir dentro del plan de desarrollo municipal proyectos de formación turística a la comunidad que se puedan llevar a cabo mediante alianzas con instituciones que presten estos servicios educativos, como iniciativa de fortalecimiento de cultura turística, manejo adecuado de los residuos contaminantes, aprovechamiento prudente al realizar estas actividades en espacios verdes como montañas, quebradas y senderos para que haya claridad en la medidas implementadas desde la administración pública.

Formular proyectos turísticos con enfoque de sostenibilidad en el banco de proyectos del municipio y de esta manera proyectar el municipio de Chinácota a través del turismo con carácter de sostenibilidad con una oferta planificada, organizada, innovadora donde el aprovechamiento de su territorio que es en un $90 \%$ verde sea el pilar y de esta manera hacerle frente a los cambios a enfrentar luego de esta pandemia, donde los destinos más valorados serán la naturaleza y turismo experiencial así estar alineados con los objetivos de 
Identificación de estrategias de gestión pública para el fomento del turismo sostenible en Chinácota, Norte de Santander

38

desarrollo del milenio garantizando la sostenibilidad del medio ambiente.

\section{Referencias}

Bertoni, Marcela (2008). Turismo sostenible: su interpretación y alcance operativo. Cuadernos de Geografía: Revista Colombiana de Geografía, (17),155-163.[fecha de Consulta 25 de Marzo de 2021]. ISSN: 0121-215X. Disponible en: https://www.redalyc.org/ articulo.oa?id=281821942010

Bernal, César Augusto. (2000)"Metodología de la investigación para Administración y Economía". Pearson Educación de Colombia. Santafé de Bogotá. Colombia. Págs. 262.

Cruz Hernández, José Gerardo (2009). GERENCIA PÚBLICA EN COLOMBIA, HACIENDO ÉNFASIS EN EL ACTUAL GOBIERNO. Entramado, 5(2),152-163.[fecha de Consulta 25 de Marzo de 2021]. ISSN: 19003803. Disponible en: https://www.redalyc.org/ articulo.oa?id=265419724010

Chávez Dagostino, Rosa María, \& Andrade Romo, Edmundo, \& Espinoza Sánchez, Rodrigo (2013). Turismo y desarrollo sustentable: contribución de Hispanoamérica. Teoría y Praxis, (13),933.[fecha de Consulta 26 de Marzo de 2021]. ISSN: . Disponible en: https://www.redalyc. org/articulo.oa? $\mathrm{id}=456145103002$

DNP (2007) Índice departamental de innovación para Colombia. https:/www.dnp.gov.co/ estudios-y-publicaciones/publicaciones/ Paginas/2017).aspx

DANE. (2018) Servicios Turismo. https://www. dane.gov.co/index.php/estadisticas-por-tema/ servicios/turismo

Guerrero, J.J., Espinosa, J.F., y Silva, L.M (2017) NORTE DE SANTANDER, COLOMBIA.
TURISMO NATURALEZA Y AVENTURA: APUESTA ESTRATÉGICA PARA LA PROYECCIÓN DEPARTAMENTAL EN EL POSCONFLICTO. International Journal of scientific Management and Tourism, 2017, Vol. $3 \mathrm{~N}^{\mathrm{o}} 1$ pp $147-177$

González-Mendoza, J. A., \& Fonseca-Vigoya, M. D. S. (2016). Cadena de Valor Turismo de Salud del Área Metropolitana de Cúcuta. Respuestas, 21(1), 28-44. https://doi. org/10.22463/0122820X.632

Moreno Alarcón, Daniela (2014). El camino del turismo con igualdad. El caso de Cartagena de Indias, Colombia. Memorias. Revista Digital de Historia y Arqueología desde el Caribe, (23),26-51.[fecha de Consulta 25 de Marzo de 2021]. ISSN: . Disponible en: https://www. redalyc.org/articulo.oa?id $=85532558003$

Matajira V., Jorge M., \& Poveda, Jesús, \& Ibáñez, Bibiana (2012). APORTES A LA CONSTRUCCION DE UNA VISION FRONTERIZA PARA LA SUBREGION SURORIENTAL DEL DEPARTAMENTO NORTE DE SANTANDER. Aldea Mundo, 17(33),31-42. [fecha de Consulta 25 de Marzo de 2021]. ISSN: 1316-6727. Disponible en: https:// www.redalyc.org/articulo.oa? $\mathrm{id}=54332636005$

Mincomercio (2018) Informes de Turismo. https:// www.mincit.gov.co/estudios-economicos/ estadisticas-e-informes/informes-de-turismo

Morillo Moreno, Marysela Coromoto (2011). Turismo y producto turístico. Evolución, conceptos, componentes y clasificación. Visión Gerencial, (1),135-158. [fecha de Consulta 25 de Marzo de 2021]. ISSN: 1317-8822. Disponible en: https://www.redalyc.org/articulo. oa? $\mathrm{id}=465545890011$

REYES VARGAS, María Victoria, \& ORTEGA OCAÑA, Ángel Fernando, \& MACHADO 
CHAVIANO, Esther Lidia (2017). MODELO PARA LA GESTIÓN INTEGRADA DEL TURISMO COMUNITARIO EN ECUADOR, CASO DE ESTUDIO PASTAZA. REVESCO. Revista de Estudios Cooperativos, (123),250-275.[fecha de Consulta 25 de Marzo de 2021]. ISSN: 11356618. Disponible en: https://www.redalyc. org/articulo.oa? id $=36750475010$

Ramirezparis Colmenares, Xiomara, \& Sepúlveda Yáñez, Álvaro Jesús (2011). Diseño de una estrategia promocionalpara posicionar la Zona Norte de Santander (Colombia) Estado Táchira (Venezuela). Pensamiento \& Gestión, (31),56-72.[fecha de Consulta 25 de Marzo de 2021]. ISSN: 1657-6276. Disponible en: https://www.redalyc.org/articulo. oa? $\mathrm{id}=64620759004$

Pardellas de Blas, Xulio X., \& Padín Fabeiro, Carmen (2004). La planificación turística sostenible: un análisis aplicado al municipio de Caldas de Reis . Revista Galega de Economía, 13(1-2),0.[fecha de Consulta 26 de Marzo de 2021]. ISSN: 1132-2799. Disponible en: https://www.redalyc.org/articulo. oa?id=39113209

UNTWO (2017) la organización mundial del turismo y la organización internacional del trabajo debaten las condiciones laborales del sector. https://www.unwto.org/es/laorganizacion-mundial-del-turismo-y-laorganizacion-internacional-del-trabajodebaten-las

Velazquez Valadez, G., \& Cardona Reséndiz, G. A. (2017). El crecimiento económico en México y su relación con el turismo y medio ambiente (2003-2015). Mundo FESC, 7(13), 56-72. Recuperado a partir de https:// www.fesc.edu.co/Revistas/OJS/index.php/ mundofesc/article/view/104 